\title{
Immune reconstitution inflammatory syndrome (IRIS) in central nervous system, due to cryptococcal infection, TB or both?
}

\author{
Dr WCJK Jayakody', Dr KGK Ubeysekara², Dr NH Kumarasinghe ${ }^{3}$
}

\begin{abstract}
Tuberculosis (TB) and cryptococcal meningitis remain the commonest causes of meningitis in patients with advanced HIV. This is a case history of an HIV patient on antiretroviral therapy (ART) for 2 months presented with CNS symptoms suggesting possible IRIS for an intra cerebral infection or any other CNS lesion. However, in diagnostic work-up patient is found to have features for tuberculosis as well as cryptococcal meningeal infection making a diagnostic dilemma of the reason for the IRIS

Key words: Immune Reconstitution Inflammatory syndrome, Cryptococcosis, TB, Central nervous system

Authors: ${ }^{1}$ Dr WCJK Jayakody (MBBS, PgDipVen, MD), Consultant Venereologist, National STD/AIDS Control Programme (NSACP), Sri Lanka. ${ }^{2}$ Dr KGK Ubeysekara (MBBS, PgDipVen, MD), Acting Venereologist, NSACP, Sri Lanka. ${ }^{3} D r ~ N . H$ Kumarasinghe, MBBS, PgDipVen, Registrar in Venereology, NSACP, Sri Lanka

Corresponding author: ${ }^{1}$ Dr WCJK Jayakody Email:wcjksovis@gmail.com Acknowledgement: All the Consultant physicians attached to Medicine professorial unit, Faculty of

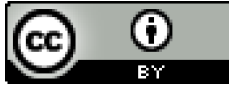
medicine, Colombo, who including the neurology team who actively involved in managing this patient.

Conflict of interest: Authors claim no conflicts of interest, Funding: not funded, Originality: This is an original work which has been not published anywhere else
\end{abstract}

\section{Full article}

\section{Introduction}

Tuberculosis and Cryptococcal meningitis are the most common causes of opportunistic meningitis in HIV infected patients and share similar clinical and laboratory features resulting in delays to diagnosis and poorer outcomes (J E Vidal, E J F Peixoto de Miranda, J Gerhardt, M Croda, \& D R Boulware, 2017). Globally, Tuberculosis remains the leading cause of death among people living with HIV, causing one in every third AIDS related deaths (AIDS, 2019, pp. 2-4). The burden of the disease is greatest in middle- and low-income countries, accounting for $95 \%$ of cases (AIDS, 2019).

\section{Case Report}

A 33-year-old, HIV seropositive male presented with intermittent fever, headache, vomiting for two weeks and altered behavior for one day to emergency medical ward. Headache was an occipital, moderate intensity and constant pain. There were several episodes of vomiting with worsening headache without visual disturbances or photophobia.
There was no slurring of speech, mouth deviation or weakness of limbs. He had been diagnosed with HIV infection 4 months back, when presented with respiratory failure due to Pneumocystis jiroveci pneumonia. His baseline CD4 count was 86 cells $/ \mathrm{mm}^{3}$ and viral load was 196,040 copies/ $\mu \mathrm{L}$. He was started on ART \{tenofovir (TDF), emtricitabine (FTC), and efavirenz (EFV)\} two months prior to this admission after excluding pulmonary tuberculosis, Cryptococcal and Toxoplasma infections. He was also on isoniazid and cotrimoxazole prophylaxis.

On admission, patient was ill looking, afebrile and not pale. There were no skin lesions or lymphadenopathy. He did not have any CNS signs other than neck stiffness. Examination of the cardiovascular system, respiratory systems and abdomen were normal.

Laboratory investigations when he presented with CNS symptoms revealed a white cell count of $13,800 / \mu \mathrm{l}, \mathrm{CRP}<6 \mathrm{mg} / \mathrm{dl}$ and ESR was 18 $\mathrm{mm} / 1$ st hour. CD4 count was 300 cells/ $\mu \mathrm{l}$ and the HIV viral load was 373 copies/ $\mu$ l. Chest $X$ 
ray and non-contrast $\mathrm{CT}$ scan of brain was normal. Contrast enhanced MRI of brain revealed multiple ring enhancing lesions were commented as multiple small tuberculomas by consultant radiologist. Cerebro-spinal-fluid analysis showed 305 lymphocytes with 31 polymorphs and 25 red blood cells. CSF sugar level was $3.3 \mathrm{mmol} / \mathrm{l}$ (RBS, $8.6 \mathrm{mmol} / \mathrm{l}$ ) and protein level, $237 \mathrm{mg} / \mathrm{dl}$. Cryptococcal antigen and India ink stain was positive in cerebrospinal-fluid, with negative TB PCR, TB culture. CMV PCR, HSV PCR, VDRL/TPPA and bacterial culture. Serum cryptococcal antigen was tested positive., Hepatitis B and Hepatitis C screening were negative.

Since the presence of lesions suggestive of tuberculomas in MRI and high protein count and low sugar level in CSF analysis, the diagnosis of CNS TB was made and antituberculosis treatment (ATT) was started with steroid cover, though there were no microbiological evidence. Even though, the CNS finding were positive for cryptococcal infection as well, initially treatment was started to cover the TB meningitis with ATT considering clinical and epidemiological grounds. Despite being on ATT, patient became clinically unstable with seizures and MRI showed expansion of lesions. The diagnosis of cryptococcal IRIS came forward due to this and subsequently amphotericin B and oral flucytosine were initiated 2 weeks after ATT continuing steroids. Following one week of amphotericin B, patient's serum creatinine levels elevated indicating an acute kidney injury with hypomagnesaemia. ART dose was adjusted according to e-GFR and some ATT were substituted to continue induction phase.

At the end of the induction phase (14 days), third MRI showed a reduction in number and intensity of lesions with clinical improvement. Repeat CSF analysis showed a marked response with 50 lymphocytes, 4 polymorphs, and a protein level of $95 \mathrm{mg} / \mathrm{dl}$. Cryptococcal antigen and India ink stain became negative. IV amphotericin was continued for a total of 21 days and followed by consolidation and maintenance phase for up to one year with ATT.

\section{Discussion}

The term immune reconstitution inflammatory syndrome (IRIS) has been used to describe a group of clinical syndromes associated with immune reconstitution that is observed commonly for mycobacterial infection, but also for other opportunistic infections (Ols), including Pneumocystis jirovecii pneumonia (PCP), toxoplasmosis, hepatitis $B$ and hepatitis $C$ infections, cytomegalovirus (CMV) infection, varicella-zoster virus (VZV) infection and cryptococcal infection (Navs E, 2002).

Cryptococcal meningitis is caused by Cryptococcus neoformans var gatti or var neoformans which is an encapsulated yeast. People get infected through inhalation and lungs get affected as the primary site (Prevention, 2018). Only in $1 / 4$ or $1 / 3$ of patients would have symptoms of meningism like neck stiffness, photophobia. Patients would express lethargy, altered mentation, personality changes, memory loss, cranial nerve abnormalities as encephalopathic symptoms (Prevention, 2018).

In this case, the development of new symptoms after two months of ART and there was rapid rise of $C D 4$ count from initial count of 86 to 300 cells/ $\mu \mathrm{l}$ and significant drop of VL from 196,040 to 373 copies $/ \mathrm{ml}$. This background was more suggestive of an IRIS for a hidden infection.

Considering the clinical presentation of CNS symptoms and signs in this patient with retroviral infection, the possible differential diagnosis would be TB-IRIS, Cryptococcal IRIS, IRIS of both infections, CNS toxoplasmosis, bacterial meningitis and viral meningitis. With regard to the very small sizes and multiplicity of the ring enhancing lesions in the MRI, negativity of toxoplasma antibodies and intake of cotrimoxazole prophylaxis were towards the exclusion of CNS toxoplasmosis. The CSF full report was not compatible with viral meningitis and at the same time CMV and HSV PCR were negative in the CSF excluding viral infection. Bacterial cultures became negative in CSF samples and the full report was not agreeable with bacterial meningitis. In fact, the most probable diagnosis for this case would be TB-IRIS, C-IRIS or IRIS due to both infections. 
Features of worsening clinical condition with ATT and merely the clinical diagnosis of TB with the supportive evidence with MRI at the beginning are more towards the diagnosis of $\mathrm{C}$ IRIS. Similarly, the positivity of India Ink stain and $\mathrm{CrAg}$ in the CSF are more confirmatory towards the C-IRIS. Although, the sensitivity and specificity of TB GeneXpert in CSF is $79.5 \%$ and $98.6 \%$ respectively and the sensitivity for CSF culture for TB is $10 \%$ to $60 \%$ (Association, 2017). According to these factors it should be either C-IRIS or IRIS of both infections. At this point, the initial diagnosis of tuberculomas in the MRI is doubtful with the possibility of cryptococcomas since they responded well following the treatment with amphotericin-B. However, considering the epidemiological ground and the validity of diagnostic tests used, prospect of delayed response to ATT cannot be excluded.

According to this case scenario, the patient was screened negative for pulmonary TB at the initiation of ART. Therefore, the adequacy of screening only for pulmonary $T B$, when the patients are more susceptible to have extrapulmonary TB during the later stages of HIV infection remains uncertain. Furthermore, with the initial negative serum $\mathrm{CrAg}$, the possibility of newly acquiring a cryptococcal infection is also a possibility with persistent immunosuppression. However, the rapid CD4 rise with ART might come against the acquisition of new infection.

IRIS may be unmasking or paradoxical. In patients diagnosed with infection during preART period, "paradoxical" IRIS occurs following ART initiation. Unmasking IRIS may arise as an emergence of previously sub-clinical disease during immune reconstitution or simply as an association with persistent immune deficiency (G. NUNNARI, 2011).

Risk factors for the development of IRIS are higher HIV-1 viral load prior to ART, low baseline CD4+ T-cell count, early commencement of ART, greater CD4 count rise in the first 6 months of ART. This is for C-IRIS are higher serum cryptococcal antigen ( $\mathrm{CrAg}$ ) titer at diagnosis and lack of initial CSF inflammation (CSF protein $<50 \mathrm{mg} / \mathrm{dL}$ and WBC
$<25$ cells $/ \mu \mathrm{L}$ ) prior to ART (Lewis J Haddow, 2010).

The treatment for Cryptococcal meningitis is amphoteracin B and flucytocin. Amphotericin $B$ deoxycholate is associated with dose-limited toxicities, most importantly, infusion-related reactions and nephrotoxicity. Amphotericin B lipid formulations are safer alternatives than conventional drug, with equivalent efficacy (Jill P Adler-Moore1, 2015). Certain people have been identified as more susceptible to develop nephrotoxicity with AmB. These include male gender, higher average daily dose of AmB ( $>$ or $=35 \mathrm{mg} /$ day), use of diuretics, body weight > or $=90 \mathrm{~kg}$, concomitant use of nephrotoxic drugs and abnormal baseline renal function (Robert A. Larsen, 2003). Our patient developed nephrotoxicity while on AmB, and that normalized following treatment completion.

There are multiple long-term complications even with adequate treatment of CNS cryptococcal disease. They might suffer mainly from residual headache (38\%), motor deficit $(15 \%)$, vertigo (15\%), and impaired cognitive function. Almost $40 \%$ of the survivors reported having some form of "disability" at 6 months and $11 \%$ of survivors declared them- selves unable to work at 1 year (Estelle Pasquier, 2018).This patient complained of residual headache on and off.

Previous studies have demonstrated that TB infection as a predisposing factor for developing cryptococcosis (Esaki Muthu Shankar, 2008). Further the diagnosis may become confusing in similar situations. Whether the IRIS is due to cryptococcal infection or duel pathology with prominent CIRIS in the background of TB, remains uncertain.

\section{References}

1. AIDS, J. U. (2019). Progress towards 2020target. In tuberculosis and HIV (pp. 2-4). Geneva: UNAIDS.

2. Association, B. H. (2017). BHIVA guidelines for the management of TB/HIV co-infection in adults.

3. Esaki Muthu Shankar, R. V. (2008). Does CD4+CD25+foxp3+ cell (Treg) and IL-10 profile determine susceptibility to immune reconstitution inflammatory syndrome (IRIS) in HIV disease? Journal of Inflammation. 
4. Estelle Pasquier, 1. J. (2018). Long-term Mortality and Disability in Cryptococcal Meningitis: A Systematic Literature Review. Clinical Infectious Diseases, 1122-1132.

5. G. NUNNARI, M. G. (2011, December). Cryptococcus-Related Immune Reconstitution Inflammatory Syndrome(IRIS): Pathogenesis and Its Clinical Implications. Curr Fungal Infect Rep.

6. J E Vidal, 1. M., E J F Peixoto de Miranda, 4. M., J Gerhardt, 5. M., M Croda, 5. M., \& D R Boulware, 6. M. (2017). Is it possible to differentiate tuberculous and cryptococcal meningitis in HIV-infected patients using only clinical and basic cerebrospinal fluid characteristics? February 2017, Vol. 107, No. 2.

7. Jill P Adler-Moore1, *. J.-P. (2015). Comparison between liposomal formulations of amphotericin $B$. Internatonal Society for human and animal mycology, 1-9.
8. Lewis J Haddow, M. P. (2010). Cryptococcal Immune Reconstitution Inflammatory Syndrome in HIV-1-infected individuals: Literature Review and Proposed Clinical Case Definitions. Lancet Infect Diseases, 10(11): 791-802.

9. Navs E, M.-D. P. (2002, December). Paradoxical rections of TB in patients with AIDS who are treated with HAART. Archives of Internal Medicine, 162:9799.

10. Prevention, C. f. (2018). Cryptococcal infection of the Central Nervous System. In Guidelines for the Prevention and Treatment of Oppertunistic infections in Adults and Adolescents with HIV. Centers for Disease Control and prevention.

11. Robert A. Larsen, 1. *. (2003). Amphotericin B and Fluconazole, a Potent Combination Therapy for Cryptococcal Meningitis. American Society for Microbiology, 385-391. 